\title{
Fatal vaccine-induced immune thrombotic thrombocytopenia (VITT) post Ad26.COV2.S: first documented case outside US
}

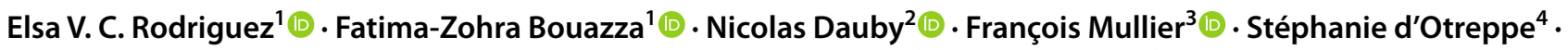

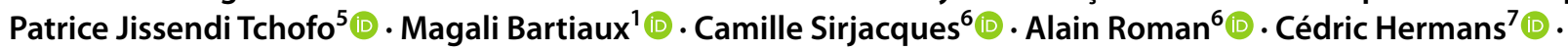 \\ Manuel Cliquennois ${ }^{8}$ (D)
}

Received: 14 June 2021 / Accepted: 1 October 2021 / Published online: 9 October 2021

(c) The Author(s), under exclusive licence to Springer-Verlag GmbH Germany 2021

\begin{abstract}
Purpose We reported the first described post Ad26.COV2.S (Janssen, Johnson \& Johnson) vaccine-induced immune thrombocytopenia (VITT) case outside US.

Case description CA young woman without any medical history presented association of deep vein thrombosis and thrombocytopenia at day 10 after vaccine injection. The patient was treated with low-molecular weight heparin at a first medical institution. Twelve days post Ad26.COV2.S vaccination, the patient was admitted at our hospital for neurological deterioration and right hemiplegia. Medical imaging using MRI showed thrombosis of the major anterior part of the sagittal superior sinus with bilateral intraparenchymal hemorrhagic complications. Screening tests for antibodies against platelet factor 4 (PF4)-heparin by rapid lateral flow immunoassay and chemiluminescence techniques were negative. Platelet activation test using heparin-induced multiple electrode aggregometry confirmed the initial clinical hypothesis. Despite immediate treatment with intravenous immunoglobulin, dexamethasone, danaparoid and attempted neurosurgery the patient evolved toward brain death.

Conclusion Even though it is an extremely rare complication of vaccination physicians should maintain a high index of suspicion of VITT in patients who received an adenovirus-vector-based SARS-CoV-2 vaccine within the last 30 days with persistent complains compatible with VITT or thromboembolic event associated with thrombocytopenia. The diagnosis should not be excluded if the rapid anti-PF4 immunological nor chemiluminescence techniques yield negative results. An adapted functional assay should be performed to confirm the diagnosis. Early treatment with intravenous immunoglobulin and non-heparin anticoagulants is essential as delayed diagnosis and administration of appropriate treatment is associated with poor prognosis.
\end{abstract}

Keywords COVID-19 · Vaccine · Ad26.COV2.S · Vaccine-induced thrombotic thrombocytopenia · VITT · Thrombosis with thrombocytopenia syndrome $\cdot$ TTS $\cdot$ Cerebral venous sinus thrombosis $\cdot$ Thrombosis

Elsa V. C. Rodriguez and Fatima-Zohra Bouazza Bouazza contributes equally as co-first authors.

Manuel Cliquennois

manuel.cliquennois@gmail.com

1 Department of Emergency Medicine, CHU Saint-Pierre, Université libre de Bruxelles (ULB), Brussels, Belgium

2 Department of Infectious Diseases, CHU Saint-Pierre, Université libre de Bruxelles (ULB), Brussels, Belgium

3 Namur Thrombosis and Hemostasis Center (NTHC), CHU UCL Namur, Université catholique de Louvain (UCL), Yvoir, Belgium

4 Department of Laboratory Medicine, LHUB-ULB, Université libre de Bruxelles (ULB), Brussels, Belgium
5 Department of Radiology, CHU Saint-Pierre, Université libre de Bruxelles (ULB), Brussels, Belgium

6 Department of Intensive Care Medicine, CHU Saint-Pierre, Université libre de Bruxelles (ULB), Brussels, Belgium

7 Division of Hematology, Hemostasis and Thrombosis Unit, Saint-Luc University Hospital, Université catholique de Louvain (UCL), Brussels, Belgium

8 Department of Clinical Hematology, CHU Saint-Pierre, Université libre de Bruxelles (ULB), Rue Haute 322, 1000 Brussels, Belgium 


\section{Introduction}

Vaccine-induced thrombotic thrombocytopenia (VITT) also described as thrombosis with thrombocytopenia syndrome (TTS) has emerged as a very rare complication of adenovector-based immunization [1-6]. VITT was initially described with the ChAdOx $1 \mathrm{nCoV}-19$ vaccine (AstraZeneca) during its deployment in Europe [3]. Recent reports from the US have highlighted the risk of VITT with the Ad26.COV2.S (Janssen, Johnson \& Johnson) [1, 7, 8]. The Ad26.COV2.S vaccine is a replication-incompetent COVID-19 vaccine requiring only one administration that has demonstrated efficacy in the prevention of symptomatic COVID-19 [9]. It has been emergency-used approved by the US Food and Drug Administration (FDA) on February 27th 2021 and received a conditional marketing authorization by the European Medicines Agency (EMA) on March 11th 2021 [7, 8].

We describe a case of extensive cerebral thrombosis associated with severe thrombocytopenia that resembled autoimmune heparin-induced thrombocytopenia in a patient who had received the Ad26.COV2.S vaccine. At our knowledge this is the first described post Ad26.COV2.S VITT case outside US.

\section{Case presentation}

A 37-year-old female patient with no previous medical history (notably no personal or familial thromboembolic event, no estrogen-progestogen contraception, no tobacco intoxication, any other vascular risk factor) received a first dose of Ad26.COV2.S vaccine at day \#0 (D0). Seven days after vaccination (D7), she consulted the emergency department of a first medical institution. Complains included headache, myalgia and fever. Hematologic results showed a normal platelet count. She was discharged after physical and biological examination.

On day \#10 (D10) post-vaccination, the patient consulted again at the same institution with persistent headaches and onset of left leg pain. Doppler ultrasound showed left popliteal vein thrombosis. SARS-CoV-2 PCR testing by nasopharyngeal swab was negative. The patient was discharged and treated with therapeutic doses of subcutaneous lowmolecular-weight heparin (LMWH) (tinzaparin subcutaneous $10.000 \mathrm{U} /$ day) and elastic compression stockings. A subsequent review of the file identified a thrombocytopenia at $25 \times 10^{9} / \mathrm{L}$ at this time.

At day \#12 post-vaccination (D12), at midnight, the patient suffered left hemicranial headache and vomiting episodes at home. During the night (4 a.m.), the patient presented with right hemiplegia and hemineglect. She was assisted by the emergency medical service. A left mydriasis was noted during transport to the hospital. A cranial computed tomography (CT) scan was immediately performed after admission to our institution's emergency department. The CT scan showed multiple bilateral intraparenchymal hemorrhagic areas. A carotid angio-CT showed no aneurysm, arteriovenous malformation or arterial thrombosis. The patient presented with altered consciousness that evolved quickly to coma. She was intubated at D12 5 a.m. and provided with mechanical ventilation.

The biological assays carried out on arrival in the emergency room at D12 showed thrombocytopenia at $50 \times 10^{9} / \mathrm{L}$, confirmed at $40 \times 10^{9} / \mathrm{L}$ on citrate, reduced prothrombin time (60\% [70-130]), low fibrinogen (1.01 g/L [1.5-4.0]), and elevated D-Dimer level (> 35,000 ng/mL). B HCG assay was negative. SARS-CoV-2 PCR testing by nasopharyngeal swab was negative. Testing for anti-PF4/heparin antibodies by rapid lateral flow immunoassay (STic Expertß HIT, Diagnostica Stago SAS, Asnières sur Seine, France) and chemiluminescence (HemosIL ${ }^{\circledR}$ AcuStar HIT-IgG(PF4-H), Instrumentation Laboratory, Bedford, MA, USA), was therefore, requested in the context of vaccination followed by thrombosis with thrombocytopenia. Both tests were negative.

Because of a high suspicion of VITT, intravenous immunoglobulin (IVIG) were initiated in the emergency room at a dose of $1 \mathrm{~g} / \mathrm{kg} /$ day (D12 post-vaccination, at 7 a.m.). A brain MRI was performed and showed a superior sagittal sinus thrombosis at the vertex (Fig. 1A) and a large intra-extra axial hemorrhagic collection with left lateral intraventricular hemorrhage (Fig. 1B), complicated by diffuse oedema (Fig. 1C). Immediately after the MRI at D12 8.30 a.m., the patient was transferred to the intensive care unit where she received intravenous therapeutic dose of danaparoid sodium as initial bolus followed by continuous infusion, and $40 \mathrm{mg}$ of intravenous dexamethasone.

Subsequently, the patient rapidly developed central diabetes insipidus (D12 1 p.m.) which required treatment with desmopressin $4 \mu \mathrm{g}$ intravenous. Bilateral fixed mydriasis appeared at D12 4 p.m. which justified the administration of $15 \mathrm{~g}$ of mannitol. Before an external ventricular shunt and intracranial pressure monitoring implementation, an $8.10^{11}$ platelets transfusion was infused. The patient was declared brain dead the day after her admission (day \#13 post-vaccination). A summary of the case presentation and management is reported in Fig. 2, illustrating the fulminant course of the illness.

Patient consent for an organ donation procedure was obtained from the family. However, due to the potential risk of transmission of VITT and after multidisciplinary consultation, decision was made not to proceed to organ donation.

As previously described [10], a functional assay was performed with a heparin-induced multiple-electrode aggregometry (HIMEA) on a multiplate analyzer (Roche) and confirmed VITT. In VITT, platelet aggregation already 


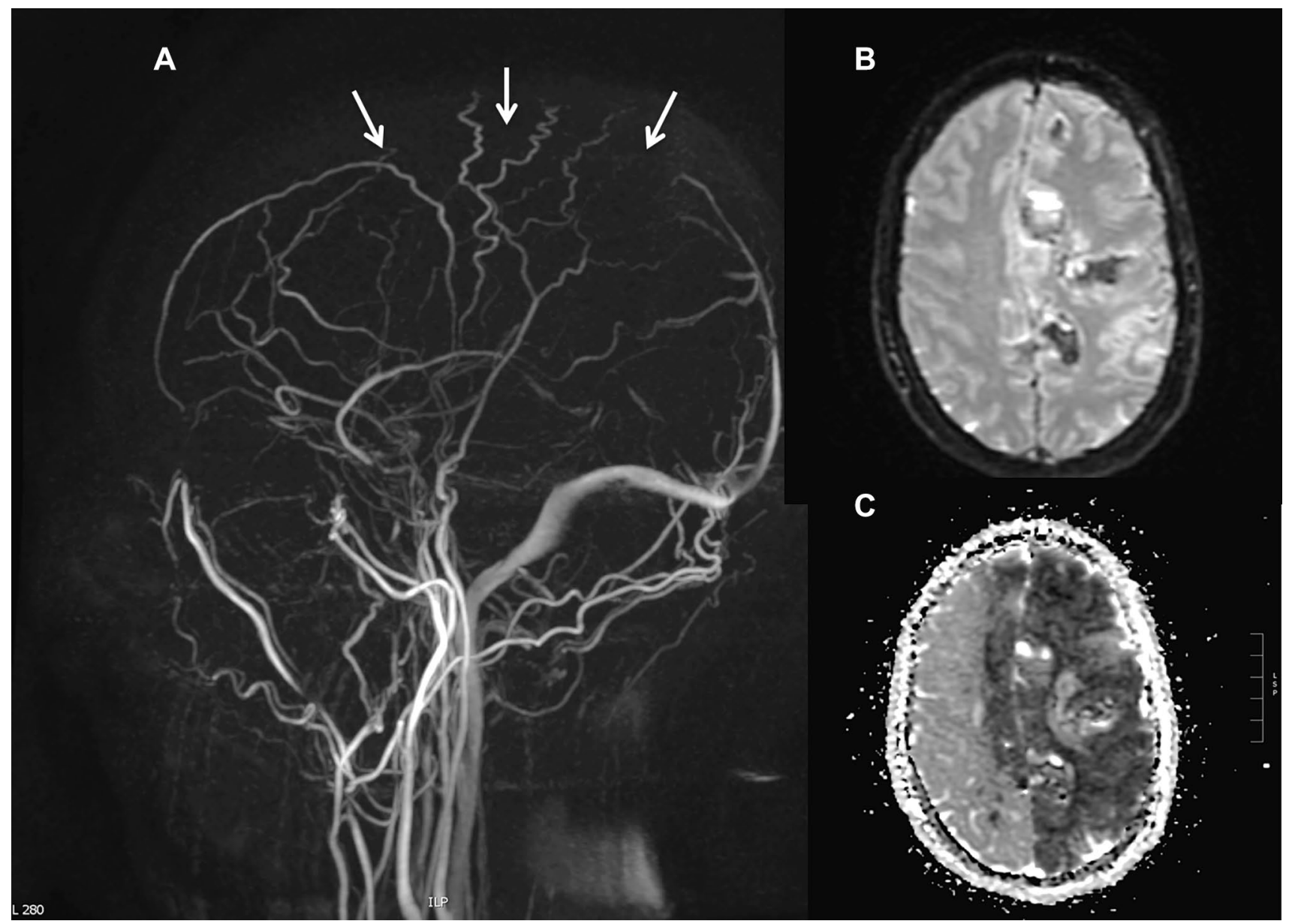

Fig. 1 Magnetic resonance imaging on day \#12 post-vaccination. The sagittal view of the 3D Phase contrast venous angiography a shows the thrombosis of the major anterior part of the sagittal superior sinus (arrows). As the consequence of it, on B0 Diffusion weighted image b multiple bilateral hemorrhagic collections are seen and on apparent diffusion coefficient map c the extensive cytotoxic oedema involves almost the whole left hemisphere and partly the right one

complication with a fulminant evolution. The interim safety data from more than 200.000 participants vaccinated with Ad26.COV2.S in South Africa were recently reported [12]. No case of VITT has been documented in the Sisonke study (ClinicalTrials.gov number, NCT04838795).

Apart from this case, all VITT cases reported in Europe involved only patients who had received the AstraZeneca adenovirus vector-based vaccine ChAdOx $1 \mathrm{nCoV}-19$. In European Union 142 cases of VITT have been reported to EudraVigilance out of 18 million ChAdOx $1 \mathrm{nCoV}-19$ vaccinated persons, as of April 13th 2021. In the United Kingdom 332 cases of VITT have been reported out of 24.2 million vaccinated persons, as of May 19th 2021. Up to May 25th 2021, The VITT cases observed with the ChAdOx 1 $\mathrm{nCoV}-19$ mainly involved young women [1]. Current guidelines in the UK recommend administering the ChAdOx 1 nCoV-19 in adults aged above 40 years old based on agedbased risk-benefit analysis. Following this first post-Ad26. COV2.S VITT case, Belgium recommended on May 26th
To our knowledge, this is the first VITT documented case outside US following Ad26.COV2.S vaccination. We offer a detailed description of a rare and life-threatening 


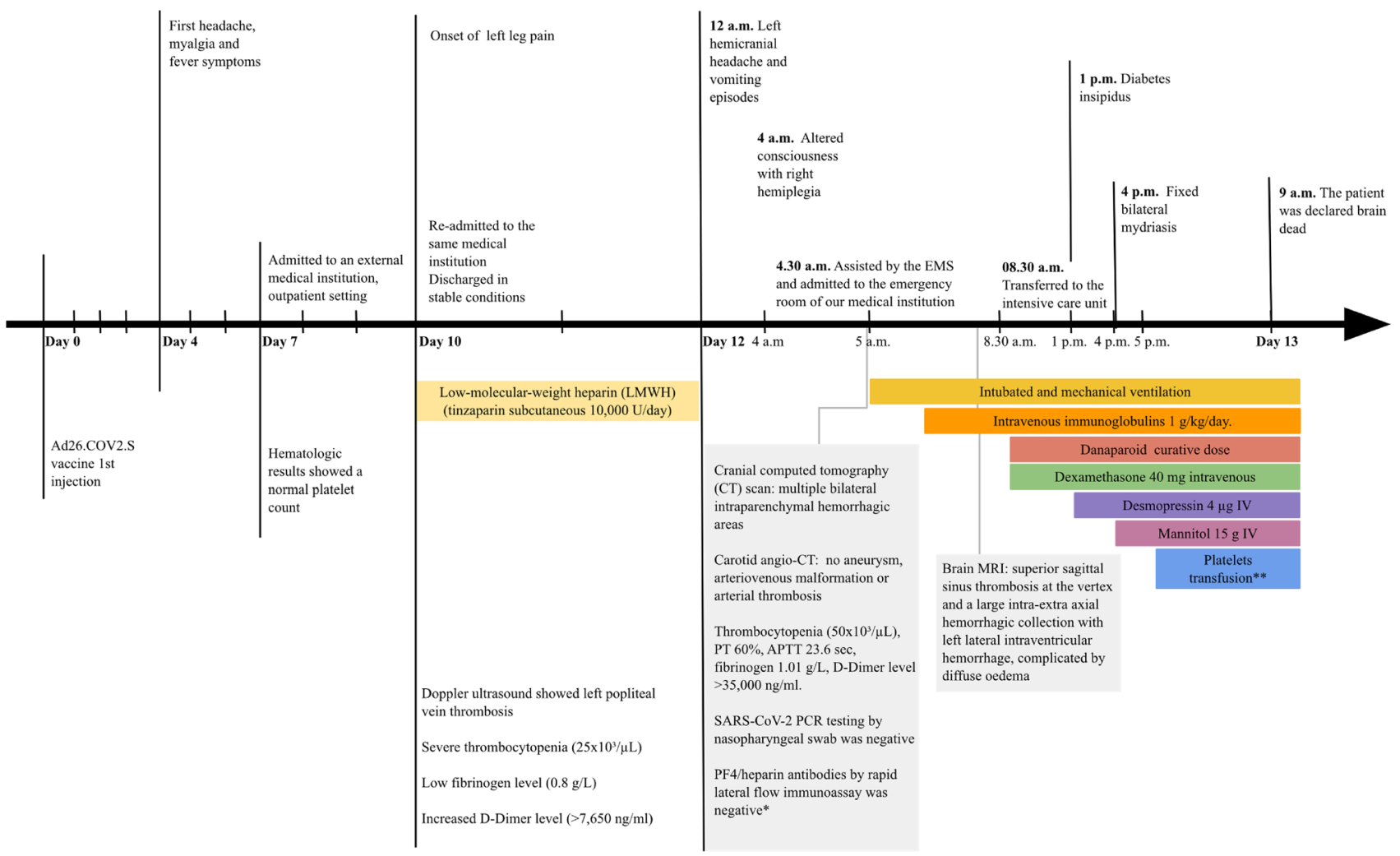

* Chemiluminescence (HemosIL $\mathbb{Q}$ AcuStar HIT-IgG(PF4-H) was also negative. On later results functional assay with a heparin induced multiple electrode aggregometry (HIMEA) was positive ** before an external ventricular shunt and intracranial pressure monitoring implementation.

PT: prothrombin time

APTT : activated partial thromboplastin time

Fig. 2 Time line summarizing the evolution, findings and management of the case

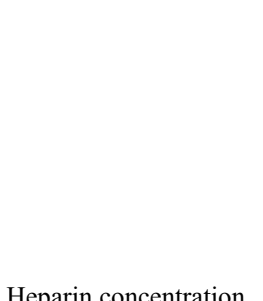

AUC

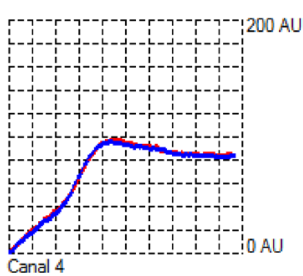

$0 \mathrm{IU} / \mathrm{mL}$

348

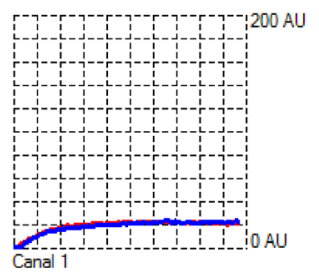

$1 \mathrm{IU} / \mathrm{mL}$

91

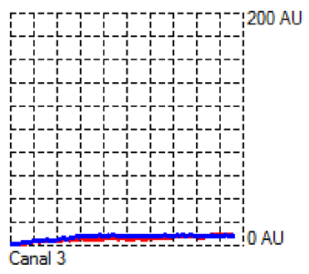

$385 \mathrm{IU} / \mathrm{mL}$

27

Fig. 3 Results of the functional assay (HIMEA): aggregation of platelets (from a good responder) after incubation with plasma from the patients was measured by whole-blood impedance aggregometry. The measurements were performed in absence of added heparin (left side)

the administration of Ad26.COV2.S only in adults aged above 41 years.

The identification of 6 VITT cases among 6.8 millions of doses in the US led to a temporary suspension of vaccination using Ad26.COV2.S on April 13th 2021. The use of Ad26. COV2.S was resumed on April 23rd 2021. FDA considered the vaccine to be safe and VITT extremely rare [7]. As of

and in the presence of low (1 IU/ml middle) or high $(385 \mathrm{IU} / \mathrm{ml}$ right side) heparin concentrations. The red and blue lines represent duplicate measurements. AU denotes arbitrary units, and AUC the area under the curve

May 7th 2021 with an exposure of 8.7 million doses administered, 28 confirmed thrombotic thrombocytopenia postAd26.COV2.S vaccine administration were reported in US. According to the FDA the patients affected by VITT after Ad26.COV2.S were only women with an average age of 37 years (range 18-59) [7]. Symptoms appeared 5-16 days post-vaccination. 
The precise mechanisms of VITT are currently unknown. However, the fact that this rare complication is only observed with adenovirus-based COVID-19 vaccine points toward an abnormal immune response to the vector $[2,13]$.

The initial clinical presentation combining headache, myalgia and fever is frequently reported and benign after COVID-19 vaccination. Unfortunately at the time of lower limb thrombosis diagnosis, the association with severe thrombocytopenia was not identified immediately as a possible VITT leading to a delay in the appropriate care. This highlights the importance of appropriate information to first-line healthcare workers and the need of a low threshold for diagnosis in case of any compatible symptoms (i.e. severe headaches) in case of recent vaccination with an adenovirus vector-based vaccine.

The results of both rapid and chemiluminescence antiPF4 assays were negative. Recent report has highlighted the low sensitivity (STic Expert HIT, one out of the 23 reported cases; Acustar, two out of the 49 reported cases) of anti-PF4 rapid immunoassays in cases of suspected VITT, including the Acustar test used in the present case $[11,14]$. This contrasts with the good performance of this assays reported in "classical" HIT [15]. Twelve US cases of cerebral venous sinus thrombosis with thrombocytopenia after Ad26.COV2.S vaccination were reported [8]. All but one (test not done) were tested positive for antiPF4 antibodies by ELISA but the type of ELISA was not provided in the report [8] despite its impact on the performance $[11,14,16]$. Only one case out of nine tested was confirmed by serotonin release assay (SRA) which illustrate that methodology should be adapted and thus provided to be sensitive to VITT [8].

Our case illustrates that clinicians should not rely on rapid anti-PF4 testing and that management should be based on the combination of hematological (thrombocytopenia) and clinical parameters in the context of recent adenovirus-vector-based COVID-19 vaccination. Rapid functional assays such as HIMEA must be carried out by an expert center quickly (in less than $24 \mathrm{~h}$ ) [17].

Current guidelines recommend against the use of unfractionated heparin or LMWH. Given the similarity between VITT and HIT's immune responses, there is a possibility that heparin may amplify platelet activation $[6,18]$. Direct-acting oral anticoagulants (DOACs) could be used in noncritically ill patients. Parenteral direct thrombin inhibitors (e.g., bivalirudin and argatroban) or danaparoid or fondaparinux are recommended, as these have been used in patients with HIT [6]. These patients should also be monitored in hospital in case of compatible symptoms.

In the setting of cerebral venous sinus thrombosis, even with hemorrhagic complication, anticoagulant therapy is recommended [19].
Intravenous immunoglobulin are considered to be standard of care of severe VITT and should be administered urgently, to reduce the platelet activation by the anti-PF4 antibodies [2, 6, 18]. Corticosteroids, like dexamethasone, should be considered, in life threatening disease and/or if IVIG administration is delayed [2,6].

The patient was considered for organ donation. This raises the issue of anti-PF4 antibody transfer to the organ transplant recipient and the risk for the graft outcome and recipient health. Previous limited experience with pretransplant HIT in donors suggest low thrombo-embolic risk in solid-organ transplant recipients $[20,21]$.

\section{Conclusion}

Physicians should maintain a high index of suspicion of VITT in patients who received an adenovirus-vector-based SARS-CoV-2 vaccine within the last 30 days with persistent complains compatible with VITT. The clinical findings of VITT are similar to HIT, but rapid immunoassays for HIT often appear as false negative in patients with VITT. The diagnosis should not be excluded if the rapid anti-PF4 immunological nor chemiluminescence techniques yield negative results. An adapted functional assay should be performed to confirm the diagnosis. For patients with active bleeding or ongoing thrombosis, an early treatment with intravenous immunoglobulin and dexamethasone is essential as delayed diagnosis and administration of appropriate treatment is associated with poor prognosis. Heparin should not be used; instead, non-heparin anticoagulants, such as bivalirudin and argatroban, danaparoid, fondaparinux or direct oral anticoagulants should be given. More research is needed on the impact pre-organ donation VITT on solid organ transplant recipient outcome.

Funding Not applicable.

Availability of data and material (data transparency) Not applicable.

Code availability (software application or custom code) Not applicable.

\section{Declarations}

Conflict of interest (include appropriate disclosures) The authors declare no conflicts of interest. ND reports personal fees from Roche and Boehringer-Ingelheim, and non-financial support from Pfizer, Janssen, and Merck Sharp \& Dohme, outside the submitted work.

Ethics approval (include appropriate approvals or waivers) Not applicable.

Consent to participate (include appropriate statements) Not applicable. 
Consent for publication (include appropriate statements) Family gives consent for publication of anonymous case report.

\section{References}

1. Muir K-L, Kallam A, Koepsell SA, Gundabolu K. Thrombotic thrombocytopenia after Ad26.COV2.S vaccination. N Engl J Med. 2021;384:1964-5. https://doi.org/10.1056/NEJMc21058 69.

2. Dotan A, Shoenfeld Y. Perspectives on vaccine induced thrombotic thrombocytopenia. J Autoimmun. 2021;121: 102663. https://doi.org/10.1016/j.jaut.2021.102663.

3. Greinacher A, Thiele T, Warkentin TE, Weisser K, Kyrle PA, Eichinger S. Thrombotic thrombocytopenia after ChAdOx 1 nCov-19 vaccination. N Engl J Med. 2021;384:2092-101. https://doi.org/10.1056/NEJMoa2104840.

4. COVID-19 vaccine (Ad26.COV2-S [recombinant]). Summary of product characteristics n.d. https://www.ema.europa.eu/en/docum ents/product-information/covid-19-vaccine-janssen-epar-productinformation_en.pdf. Accessed 9 Aug 2021.

5. Cines DB, Bussel JB. SARS-CoV-2 vaccine-induced immune thrombotic thrombocytopenia. N Engl J Med. 2021;384:2254-6. https://doi.org/10.1056/NEJMe2106315.

6. World Health Organization. Guidance for clinical case management of thrombosis with thrombocytopenia syndrome (TTS) following vaccination to prevent coronavirus disease (COVID-19) 2021. https://www.who.int/publications/i/item/WHO-2019-nCoVTTS-2021.1. Accessed 9 Aug 2021.

7. MacNeil JR, Su JR, Broder KR, Guh AY, Gargano JW, Wallace M, et al. Updated recommendations from the advisory committee on immunization practices for use of the Janssen (Johnson \& Johnson) COVID-19 Vaccine After reports of thrombosis with thrombocytopenia syndrome among vaccine recipients-United States, April 2021. MMWR Morb Mortal Wkly Rep. 2021;70:651-6. https://doi.org/10.15585/mmwr.mm7017e4.

8. See I, Su JR, Lale A, Woo EJ, Guh AY, Shimabukuro TT, et al. US case reports of cerebral venous sinus thrombosis with thrombocytopenia after Ad26.COV2.S vaccination, March 2 to April 21, 2021. JAMA. 2021. https://doi.org/10.1001/jama.2021.7517.

9. Sadoff J, Gray G, Vandebosch A, Cárdenas V, Shukarev G, Grinsztejn B, et al. Safety and efficacy of single-dose Ad26.COV2.S vaccine against Covid-19. N Engl J Med. 2021. https://doi.org/10. 1056/NEJMoa2101544.

10. Morel-Kopp M-C, Mullier F, Gkalea V, Bakchoul T, Minet V, Elalamy I, et al. Heparin-induced multi-electrode aggregometry method for heparin-induced thrombocytopenia testing: communication from the SSC of the ISTH. J Thromb Haemost. 2016;14:2548-52. https://doi.org/10.1111/jth.13516.

11. Vayne C, Rollin J, Gruel Y, Pouplard C, Galinat H, Huet O, et al. PF4 immunoassays in vaccine-induced thrombotic thrombocytopenia. N Engl J Med. 2021. https://doi.org/10.1056/NEJMc21063 83.

12. Takuva S, Takalani A, Garrett N, Goga A, Peter J, Louw V, et al. Thromboembolic events in the South African Ad26.COV2.S vaccine study. N Engl J Med. 2021;385:570-1. https://doi.org/10. 1056/NEJMc2107920.

13. Douxfils J, Favresse J, Dogné J-M, Lecompte T, Susen S, Cordonnier $\mathrm{C}$, et al. Hypotheses behind the very rare cases of thrombosis with thrombocytopenia syndrome after SARS-CoV-2 vaccination. Thromb Res. 2021;203:163-71. https://doi.org/10.1016/j.throm res.2021.05.010.

14. Platton S, Bartlett A, MacCallum P, Makris M, McDonald V, Singh D, et al. Evaluation of laboratory assays for anti-platelet factor 4 antibodies after ChAdOx1 nCOV-19 vaccination. J Thromb Haemost. 2021. https://doi.org/10.1111/jth.15362.

15. Warkentin TE, Sheppard JA-I, Linkins L-A, Arnold DM, Nazy I. High sensitivity and specificity of an automated IgG-specific chemiluminescence immunoassay for diagnosis of HIT. Blood. 2018;132:1345-9. https://doi.org/10.1182/blood-2018-04-847483.

16. Scully M, Singh D, Lown R, Poles A, Solomon T, Levi M, et al. Pathologic antibodies to platelet factor 4 after ChAdOx $1 \mathrm{nCoV}-19$ vaccination. N Engl J Med. 2021. https://doi.org/10.1056/NEJMo a2105385.

17. Elalamy I, Gerotziafas G, Alamowitch S, Laroche J-P, van Dreden P, Ageno W, et al. SARS-CoV-2 vaccine and thrombosis: expert opinions. Thromb Haemost. 2021. https://doi.org/10. 1055/a-1499-0119.

18. McCrae KR. Thrombotic thrombocytopenia due to SARS-CoV-2 vaccination. Cleve Clin J Med. 2021. https://doi.org/10.3949/ ccjm.88a.ccc078.

19. Ropper AH, Klein JP. Cerebral venous thrombosis. N Engl J Med. 2021;385:59-64. https://doi.org/10.1056/NEJMra2106545.

20. Assfalg V, Hüser N. Heparin-induced thrombocytopenia in solid organ transplant recipients: the current scientific knowledge. World J Transplant. 2016;6:165. https://doi.org/10.5500/wjt.v6. i1.165.

21. NHS Blood and Transplant. Organ donation and transplantation from patients with vaccine induced thrombosis and thrombocytopenia (VITT) n.d. https://nhsbtdbe.blob.core.windows.net/umbra co-assets-corp/23602/inf1569.pdf. Accessed 9 Aug 2021. 